\title{
RECENT BEAM STUDIES OF THE POP FFAG PROTON SYNCHROTRON
}

\author{
M. Yoshimoto, T. Adachi, M. Aiba, K. Koba, S. Machida, Y. Mori, \\ R. Muramatsu, C. Ohmori, I. Sakai, Y. Sato, M. Sugaya, A. Takagi, \\ R. Ueno, M. Wada T. Yokoi, M. Yoshii, Y. Yuasa, KEK, Ibaraki, Japan
}

\section{Abstract}

The PoP(Proof of Principle) FFAG accelerator, which was the world first FFAG proton synchrotron, was constructed at KEK. After the first proton beam acceleration of FFAG, we studied various aspects of the PoP FFAG. The observed beam orbit shift during the acceleration was consistent with the simulation result. It was confirmed that the beam intensity increased about five times with multi-bunch injection. The horizontal acceptance of the PoP FFAG was estimated at about $4000 \pi \mathrm{mm} . \mathrm{mrad}$ experimentally.

\section{INTRODUCTION}

The Fixed Field Alternating Gradient (FFAG) accelerator was proposed by Ohkawa in 1953[1]. Since then the electron FFAG accelerator was constructed and tested in MURA project[2][3]. In order to investigate a FFAG for high intensity proton accelerator, the PoP(Proof of Principle) FFAG synchrotron was constructed at KEK in March 2000[4]. That is the world first proton accelerator.

Up to now, we have been carrying out the beam experiments with the PoP FFAG to investigate the characteristics of the accelerator. In this paper, the results of the beam studies since last year, such as the RF acceleration, the multi-bunch injection, and the horizontal acceptance survey, are reported.

\section{HARDWARE DESCRIPTION}

\subsection{Beam Injection Scheme}

In the PoP FFAG, the proton beam is injected into the ring with an electro-static septum and a pair of electro-static bumps [4]. By changing the decay time of the bump voltage, two modes of the beam injection could be selected. One mode of the operation is that the decay time is set to be slower than the revolution period, so called the slow bumping mode. The electric field of the bump electrodes lasts for a few turns, as a result the bump orbit gradually shifts towards the closed orbit. Thus, multi-bunch injection could be realized through the phase space painting. The other mode of the operation is that the decay time is set nearly equal the revolution period, so called the fast bumping mode. The injected beam experiences the bump field only in the first turn, it means the bump works as a fast kicker.

\subsection{Beam Position Monitor}

The horizontal and vertical positions of the circulating beams can be measured with the beam position monitor(BPM). In the FFAG, the BPM have wide aperture, because the beam orbit shifts during the acceleration in fixed magnetic field. Figure 1-(a) shows the schematic diagram of the BPM installed in the PoP FFAG. The BPM consists of a pair of electro-static pick-ups, and the dimension of the BPM is $490 \mathrm{~mm}$ (width) x $50 \mathrm{~mm}$ (length) $\times 119 \mathrm{~mm}$ (height). When the beam passes through the BPM, it induces electric charge on the electrodes. The beam position is calculated by these two picked-up signals[5].
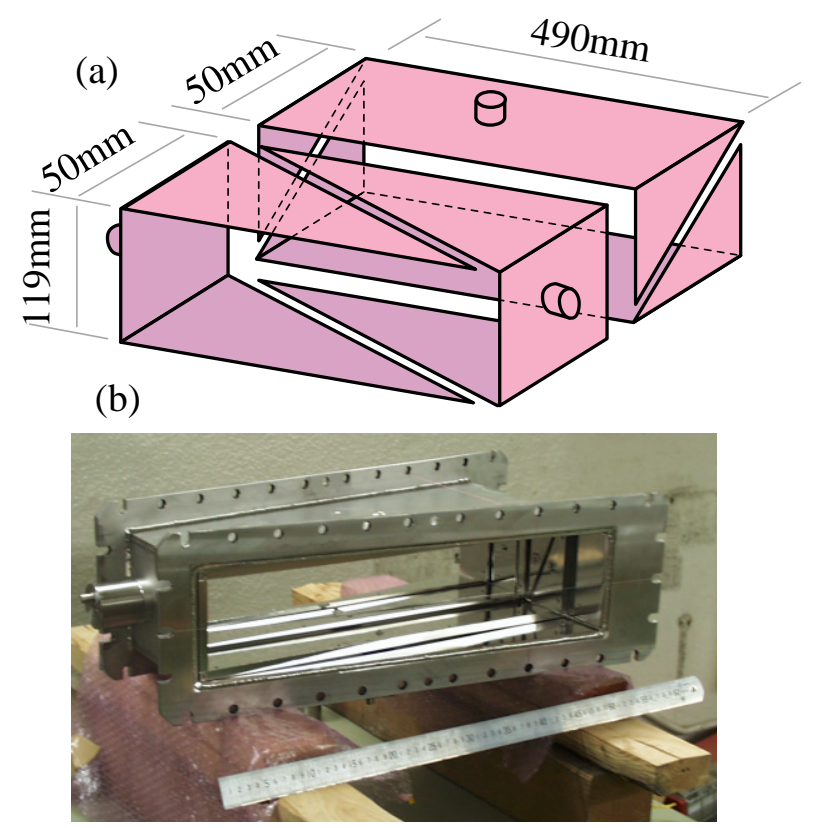

Figure 1: Beam Position Monitor (BPM).

(a) Schematic diagram of the BPM installed in the PoP FFAG.

(b) Photograph of the BPM

\section{EXPERIMENTS}

\subsection{RF Acceleration Study}

During the beam acceleration, the orbit is shifted radially in the ring. Figure 2 shows a typical result of the beam orbit shift measured with the BPM. The beam was accelerated from $50 \mathrm{keV}$ to $500 \mathrm{keV}$ within $1 \mathrm{msec}$, accordingly the beam position shifted from $765 \mathrm{~mm}$ to $1050 \mathrm{~mm}$. The results are consistent with the simulation.

In addition, by changing the final energy, the revolution frequency and synchrotron frequency were measured in each flat top. The measured both frequencies agreed with the calculated values. 


\subsection{Multi-bunch Injection Study}

As described in the section of the injection scheme, the beam intensity can be increased by the phase space painting in the slow bumping mode. Figures $4-(a)$ and (b) show typical results of the observed circulating beam signals for one-bunch injection and five-bunch injection, respectively. In this study, the decay time of the bump voltage was set $20 \mu \mathrm{sec}$. It was ascertain that the observed beam intensity increased about five times with the multi-bunch injection.

\subsection{Acceptance Survey Study}

The horizontal beam acceptance at injection could be surveyed with the fast bumping mode and one bunch injection.

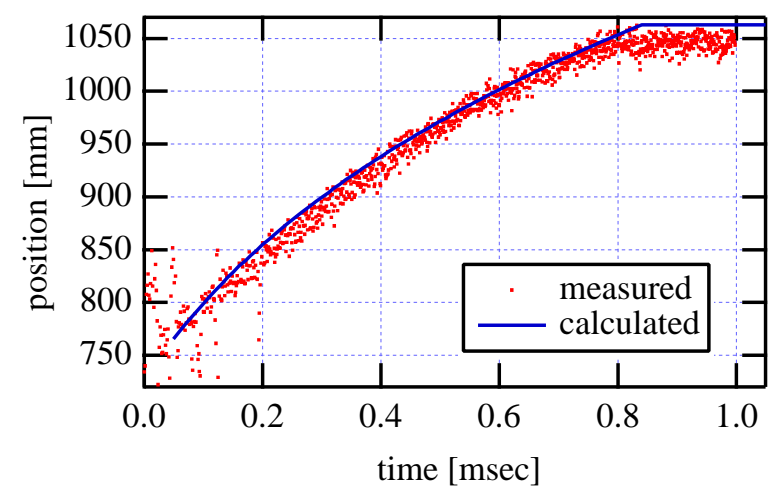

Figure 2: Beam orbit shift during the acceleration from $50 \mathrm{keV}$ to $500 \mathrm{keV}$.

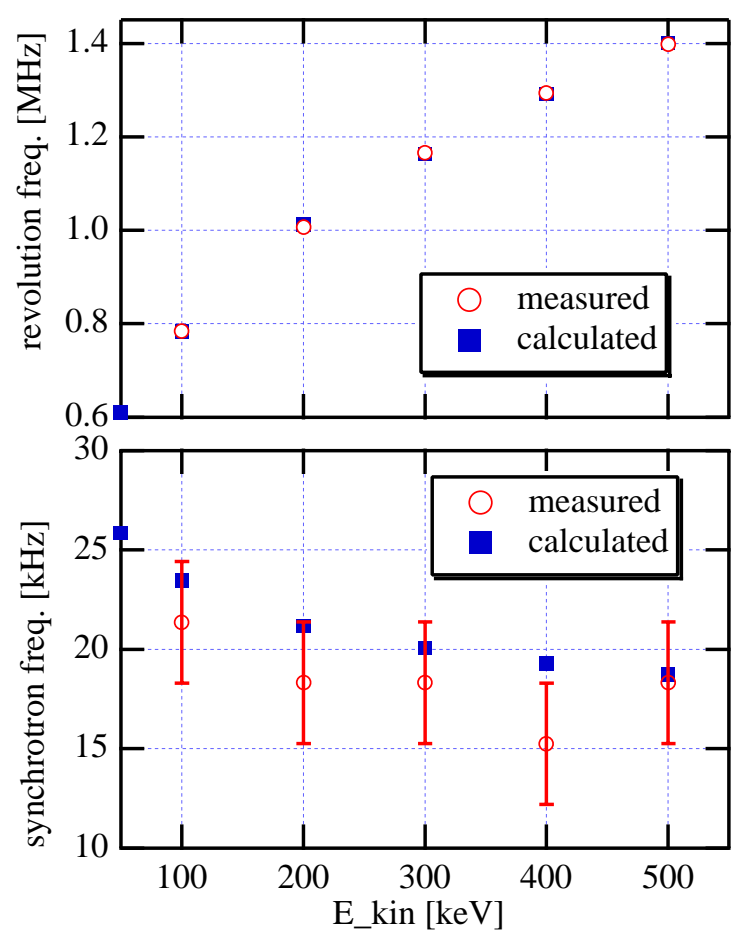

Figure 3: Revolution and synchrotron frequency in various final energies. The error bars of the bottom graph are the resolution of FFT.
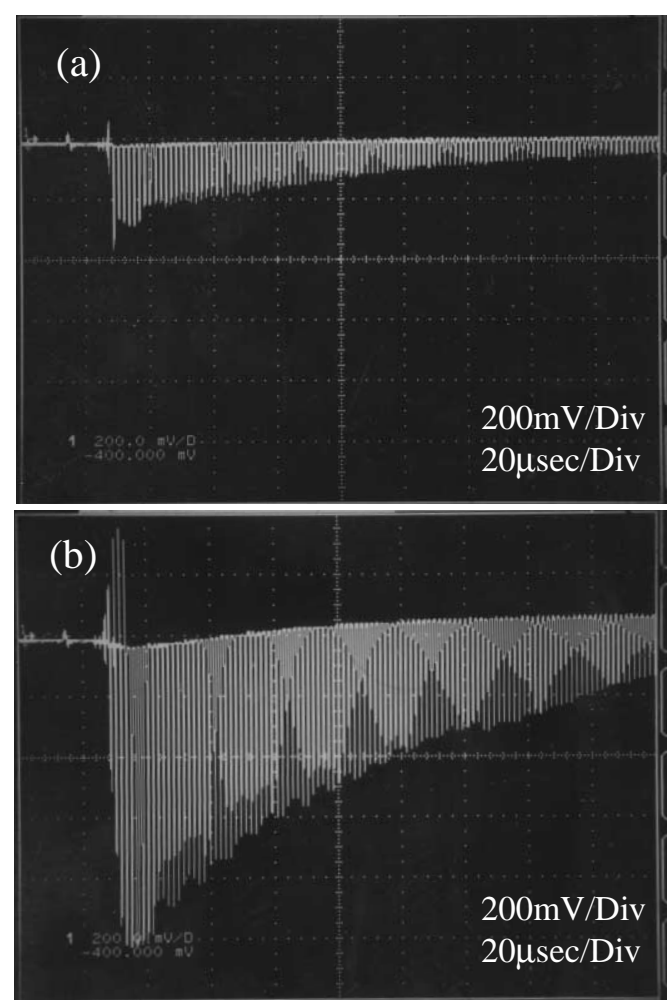

Figure 4: Typical circulating beam signal.

(a) 1 bunch injection.

(b) 5 bunch injection.

At first, the betatron oscillations were measured for various bump voltage, and the results were shown in figure 5. The amplitude of betatron oscillation was changed by varying the bump voltage. The decay time of the bump voltage was set $3 \mu \mathrm{sec}$, and bump voltage was changed from $3 \mathrm{kV}$ to $15 \mathrm{kV}$. The beam hit the septum electrode where the bump voltage was less than $3 \mathrm{kV}$, and was lost. The beam injected into the center of the phase space at $12 \mathrm{kV}$ of bump voltage, and the amplitude of betatron oscillation is the maximum at $3 \mathrm{kV}$.

Secondly, the horizontal beam acceptance was studied. The amplitude of betatron oscillation ( $\mathrm{x}$ ) can be estimated from figure 5 , and the maximum beam angle $\left(\mathrm{x}^{\prime}\right)$ at the BPM can be also estimated by the bump voltage itself. The observed sets of $\left(x, x^{\prime}\right)$ were plotted on the phase space, which was shown in figure 6 . It shows that the horizontal beam acceptance could be estimated at about $4000 \pi \mathrm{mm} . \mathrm{mrad}$ in maximum, where the shape of the acceptance was assumed to be the ellipse. The beam tracking simulation was done, which adopted the Runge-Kutta method with the magnetic field map calculated by TOSCA[4], and the result was also shown in figure 6. It gives the horizontal beam dynamic aperture to be estimated at about $10000 \pi \mathrm{mm}$.mrad. But the time [ $\mu \mathrm{sec}]$ horizontal beam aperture was limited actually by the wall of the septum which was set $40 \mathrm{~mm}$ inside of the closed orbit of the injection energy, so that the physical aperture was estimated at about $4000 \pi \mathrm{mm} . \mathrm{mrad}$. As a result, the measured horizontal beam acceptance could be explained by the physical aperture by the simulation. 


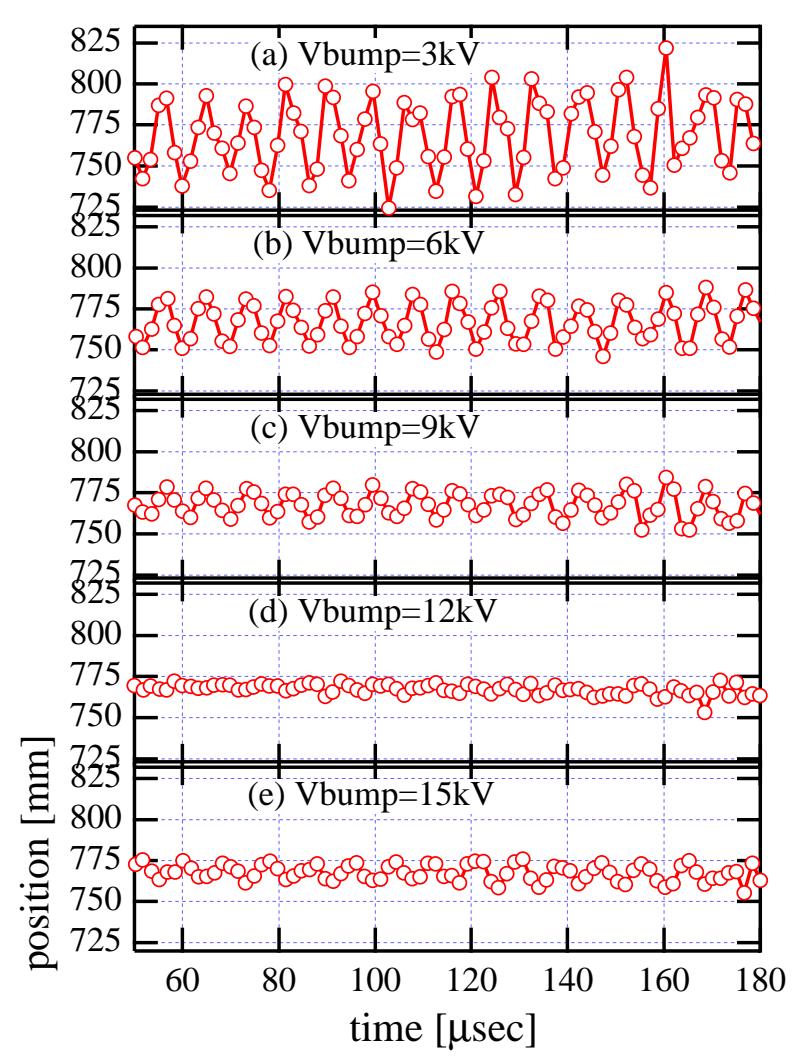

Figure 5: Betatron oscillations observed by the BPM in various bump voltage.

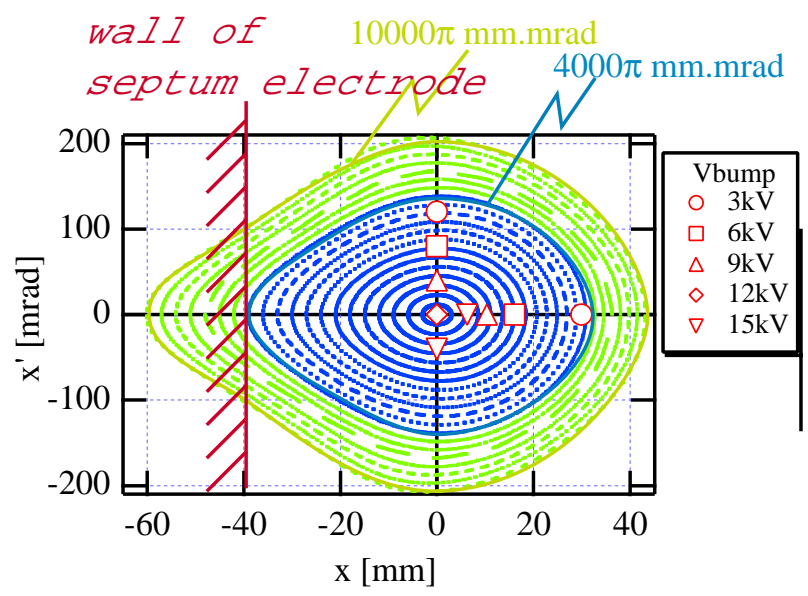

Figure 6: Horizontal beam acceptance. Markers are the measured amplitude of betatron oscillation and the expected bump kick angle. Dots are the dynamic aperture calculated by beam tracking simulation. A diagonal line is the position of the septum

\section{SUMMARY}

Beam studies of the PoP FFAG have been carried out. The beam orbit which was measured with the BPM shifted from $765 \mathrm{~mm}$ to $1050 \mathrm{~mm}$ during the acceleration and it was consistent with the simulation. The beam intensity increased about five times with five bunch injection. The measured horizontal acceptance at injection was about $4000 \pi \mathrm{mm} . \mathrm{mrad}$ which was limited by the septum electrode. All of the results indicates that the PoP FFAG accelerator works as expected.

\section{REFERENCES}

[1] C.Ohkawa, Proceedings of annual meeting of JPS(1953)

[2] K.R.Symon et al., Phys.Rev.103(1956) 1837 [3] A.A.Dolomensky et al., ZhETF 33(1957)298

[4] M.Aiba et al., Proceedings of EPAC, 2000

[5] S-i. Kurokawa, S.Y.Lee et al., Beam Measurement p270 